\title{
ARTICLE
}

Translational Therapeutics

\section{Clinical, histological and molecular predictors of metastatic melanoma responses to anti-PD-1 immunotherapy}

\author{
Frantz Dupuis ${ }^{1}$, Laurence Lamant ${ }^{2,3}$, Emilie Gerard ${ }^{4}$, Nouritza Torossiann ${ }^{5}$, Leonor Chaltiel ${ }^{6}$, Thomas Filleron ${ }^{6}$, Marie Beylot-Barry ${ }^{4,7}$, \\ Caroline Dutriaux ${ }^{4}$, Sorilla Prey ${ }^{4}$, Audrey Gros ${ }^{7,8}$, Marie-Laure Jullie ${ }^{1}$, Nicolas Meyer ${ }^{5}$ and Béatrice Vergier ${ }^{1,7}$
}

BACKGROUND: Prescribing anti-programmed death-1 (PD-1) immunotherapy for advanced melanoma is currently not restricted by any biomarker assessment. Determination of programmed death-ligand-1 (PD-L1)-expression status is technically challenging and is not mandatory, because negative tumours also achieve therapeutic responses. However, reproducible biomarkers predictive of a response to anti-PD-1 therapy could contribute to improving therapeutic decision-making.

METHODS: This retrospective study on 70 metastatic melanoma patients was undertaken to evaluate the relationships between clinical, histological, immunohistochemical and/or molecular criteria, and the 6-month objective response rate.

RESULTS: Better objective response rates were associated with metachronous metastases $(P=0.04)$, PD-L1 tumour- and/or immune-cell status $(P=0.01), C D 163+$ histiocytes at advancing edges $(P=0.009)$ of primary melanomas and NRAS mutation $(P=$ 0.019). Moreover, $C D 163+$ histiocytes at advancing edges $(P=0.04)$ were associated with longer progression-free survival (PFS), and metachronous metastases with longer overall survival $(P=0.02)$ and PFS $(P=0.049)$.

CONCLUSIONS: Combining these reproducible biomarkers could help improve therapeutic decision-making for patients with progressive disease.

British Journal of Cancer (2018) 119:193-199; https://doi.org/10.1038/s41416-018-0168-9

\section{INTRODUCTION}

Melanomas are highly immunogenic cancers, ${ }^{1}$ whose prognoses have been dramatically improved by immunotherapy. Nivolumab or pembrolizumab anti-programmed death-1 (PD-1) monoclonal antibodies have been reported to significantly increase advanced melanoma patients' response rates, progression-free survival (PFS) and overall survival (OS), compared to anti-cytotoxic T-lymphocyte antigen-4 antibodies and/or chemotherapy. ${ }^{2-5}$ Overall response rates (ORRs) in pivotal clinical studies on nivolumab or pembrolizumab, respectively, were $28 \%{ }^{6}$ or $38 \% .^{7}$ Anti-PD-1 is currently indicated as first-line treatment for BRAF- ( $\mathrm{v}$-Raf murine sarcoma viral oncogene homolog $B$ ) metastatic melanomas or sometimes $B R A F+$ ones with few metastases or slow evolution.

Tumour-cell programmed death-ligand-1 (PD-L1) expression has been associated with better outcomes under anti-PD-1 immunotherapy: a recent meta-analysis highlighted better odds ratios of ORRs for the 1\%- and 5\%-positive tumour-cell thresholds (respectively, 2.81, $P=0.0002$; and 2.22, $P<0.00001) .^{8}$ Moreover, because all studies reported significant response rates of PD-L1melanomas, ${ }^{9}$ PD-L1-status determination is not mandatory for the prescription of these agents to melanoma patients, unlike lung adenocarcinomas. ${ }^{10}$

Biological and technical challenges in immunohistochemically evaluating PD-L1 expression might explain, at least partially, PD-L1 status-therapeutic response discordances, ${ }^{11}$ because tumour and immune cells must be considered, and the latter are frequently PD-L1+. ${ }^{12}$ Moreover, other cells, e.g. CD8+ tumour-infiltrating T lymphocytes (TILs) ${ }^{13}$ or CD163+ histiocytes, ${ }^{14}$ that play important roles immune-response regulation, could be involved in immunotherapy efficacy.

We hypothesised that PD-L1-expression status combined with other clinical, histological and/or molecular criteria might be able to better predict responses to anti-PD-1 therapy than PD-L1 status alone. Therefore, we retrospectively analysed characteristics of 70 anti-PD-1-treated, metastatic melanoma patients, to try to identify markers contributive to therapeutic decision-making.

\section{MATERIALS AND METHODS}

This two-centre (Toulouse and Bordeaux), retrospective, biomarker analysis concerned a cohort receiving routine care. Seventy patients with advanced and/or metastatic cutaneous melanoma and available formalin-fixed, paraffin-embedded (FFPE) tumour blocks were identified. All primary and/or metastatic lesion specimens were collected before any antiPD-1 administration. All patients provided written informed consent for the use of the samples, and the local Ethics Committee approved the study. Clinical and histological

\footnotetext{
${ }^{1}$ Department of Pathology, Hôpital Haut-Lévêque (CHU de Bordeaux), 33604 Pessac, France; ${ }^{2}$ Department of Pathology, Oncopole de Toulouse, 31100 Toulouse, France; ${ }^{3}$ Université Paul-Sabatier, 31400 Toulouse, France; ${ }^{4}$ Department of Dermatology, Hôpital Saint-André (CHU de Bordeaux), 33000 Bordeaux, France; ${ }^{5}$ Department of Dermatology, Paul-

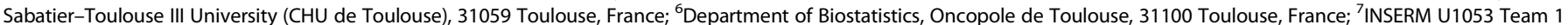
(université de Bordeaux), 33076 Bordeaux, France and ${ }^{8}$ Department of Tumour Biology and Tumour Bank, Hôpital Haut-Lévêque (CHU de Bordeaux), 33604 Pessac, France Correspondence: Béatrice Vergier (beatrice.vergier@chu-bordeaux.fr)
} 


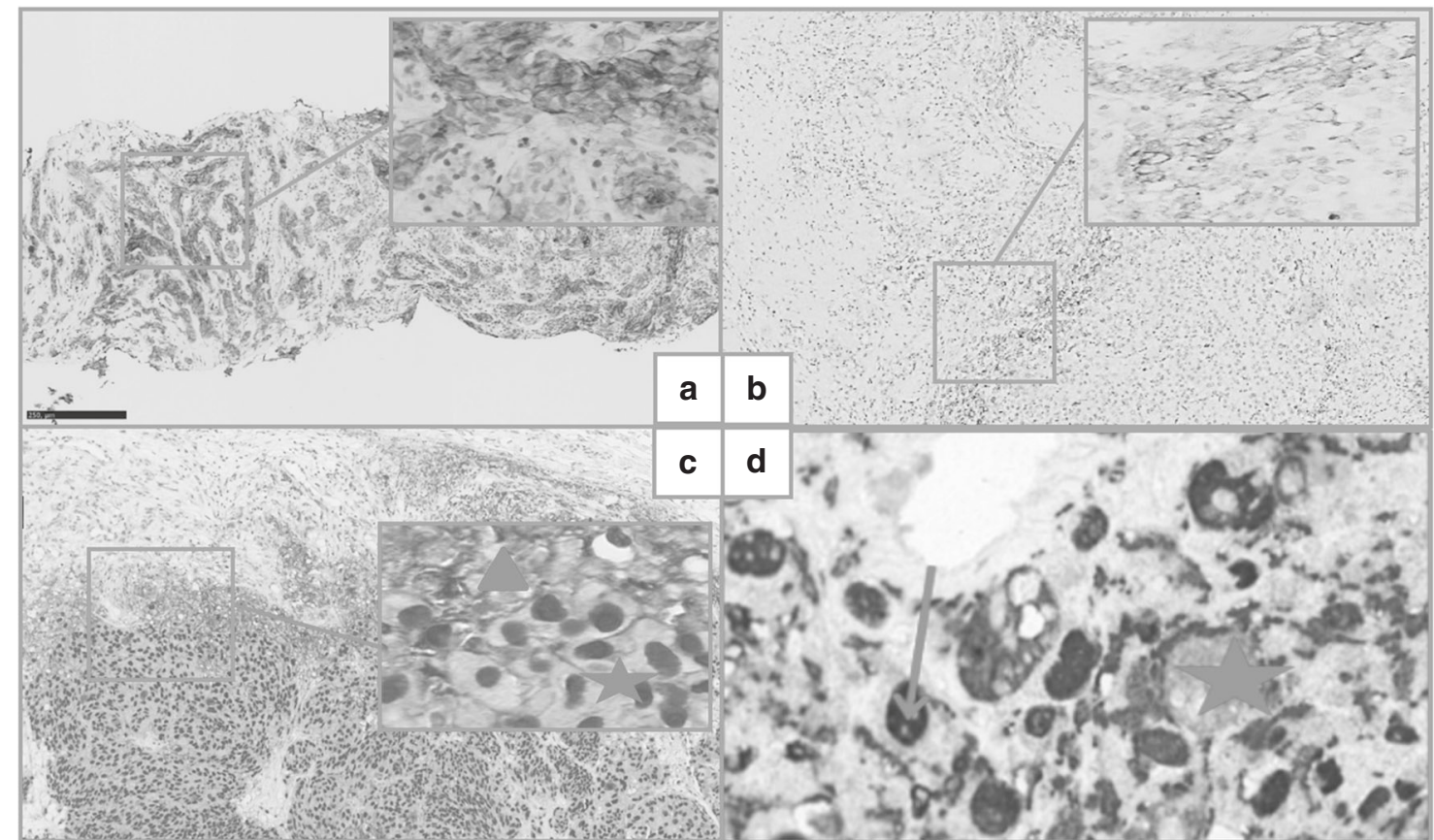

Fig. 1 Immunohistochemical labeling of metastatic melanoma primary lesions or metastases. a All tumour (melanoma) cells are programmed death-ligand-1 (PD-L1)-positive, which is quite rare ( $\times 100$, E1L3N clone). b A sample with $>5 \%$ PD-L1-positive tumour area. Note: the disposition of the PDL1+ cells at the tumour edge is very common ( $\times 100$, E1L3N clone). c SOX10 (purple nuclei) and PDL1 (brown membranes) double-labeling. Note: PD-L1+ is expressed both by immune cells (SOX10 negative, red triangle) and tumour melanocytes (SOX10 positive, red star) (×200). d SOX10 (purple nuclei, red arrow) and CD163 (brown membranes, red star) doublelabeling. Note: CD163labeling of histiocyte cytoplasmic extensions surrounding SOX10+ tumour cells could suggest that some PD-L1+ cells thought to be tumoural might rather be PD-L1- tumour cells surrounded by positive histiocyte cytoplasmic extensions $(\times 200)$

parameters and follow-up data were retrieved from medical files. The primary outcome measure was the 6-month ORR, determined radiologically with iRECIST criteria. ${ }^{15}$ We also collected acute (first month) and chronic (thereafter) immune adverse events (IAEs), defined as all AEs, most frequently skin, bowel, liver or endocrine symptoms potentially attributable to immunotherapy.

\section{Immunohistochemistry}

An automated Leica immunohistochemistry instrument labeled 3$\mu$ m-thick sections of FFPE blocks with E1L3N (Cell Signaling Technology, Leiden, The Netherlands; diluted 1:100). As stipulated in REMARK criteria, for each case, anti-PD-L1-antibody 22C3immunolabeling (Dako kit, Glostrup, Denmark) was run in parallel on an automated Dako immunohistochemistry apparatus, for comparison with the more novel E1L3N.

The percentages of PD-L1+ tumour cells and tumour-area (including tumour and/or immune cells), and PD-L1+ cells and CD163+ (clone 10D6, Leica) histiocytes at advancing edges (immediate tumour periphery) of invasive melanomas, were determined as previously described. ${ }^{8,16}$ Positivity was defined as PD-L1 cell-membrane labeling of $>5 \%$ of tumour area and cells, and $>10 \%$ of PD-L1 and CD163+ histiocytes at advancing edges (Fig. 1). The advancing tumour edge was defined as the tumour cell-peritumour-inflammation interface, without specifying width. Those values were evaluated semi-quantitatively, by light microscopy of the whole slide, not only a region, first assessing PD-L1-labeling intensity with four-tier grading $(+$ to +++$)$, but, because intensity of the vast majority of samples did not vary, we chose not to use it. PD-L1 cut-offs were chosen based on the literature findings, and those for CD163 were derived from our pathologists' preliminary study of 10 characteristic cases.
PD-L1 expressions in each patient's primary melanoma and metastases were compared. TILs, their intratumoural or peritumoural distribution pattern (brisk, with T cells throughout the lesion or at its outer edge, or non-brisk, not involving the entire lesion or outer edge) and CD8 (clone 144B, Dako) expression were analysed according to the literature. ${ }^{17,18}$ All criteria were independently assessed by three experienced dermatopathologists (FD, BV, LL) blinded to clinical-radiological information. Between-observer scoring mismatches were resolved by case review.

\section{Statistical analyses}

Categorical variables, expressed as numbers, (\%) and continuous variables, as median [range], were compared respectively using $X^{2}$ or Fisher's exact tests and Kruskal-Wallis tests. All survival parameters were measured from the date of the first anti-PD-1 infusion. Kaplan-Meier analyses of PFS and OS used the following first-event definitions, respectively: progression or death, and death. Univariate analyses used the log-rank test, with $P \leq 0.05$ defining significance. Statistical analyses were computed using STATA and R software. Given the exploratory nature of this study, no adjustments were made for multiple comparisons.

Via recursive partitioning (rpart package in $\mathrm{R}$ v4.1-10), classification-and-regression trees created a decision tree indicating how well different variables predict 6-month objective response class (confusion matrix available in supplementary figure 9). The information index was used for the splitting rule to stratify data into subsets of individuals, represented as nodes in the decision tree, with the package's default option imputing missing values. Positive- (PPV) and negative-predictive values (NPV) were calculated.

The intraclass-correlation coefficient (ICC) assessed interobserver variability of PD-L1-expression scores. 


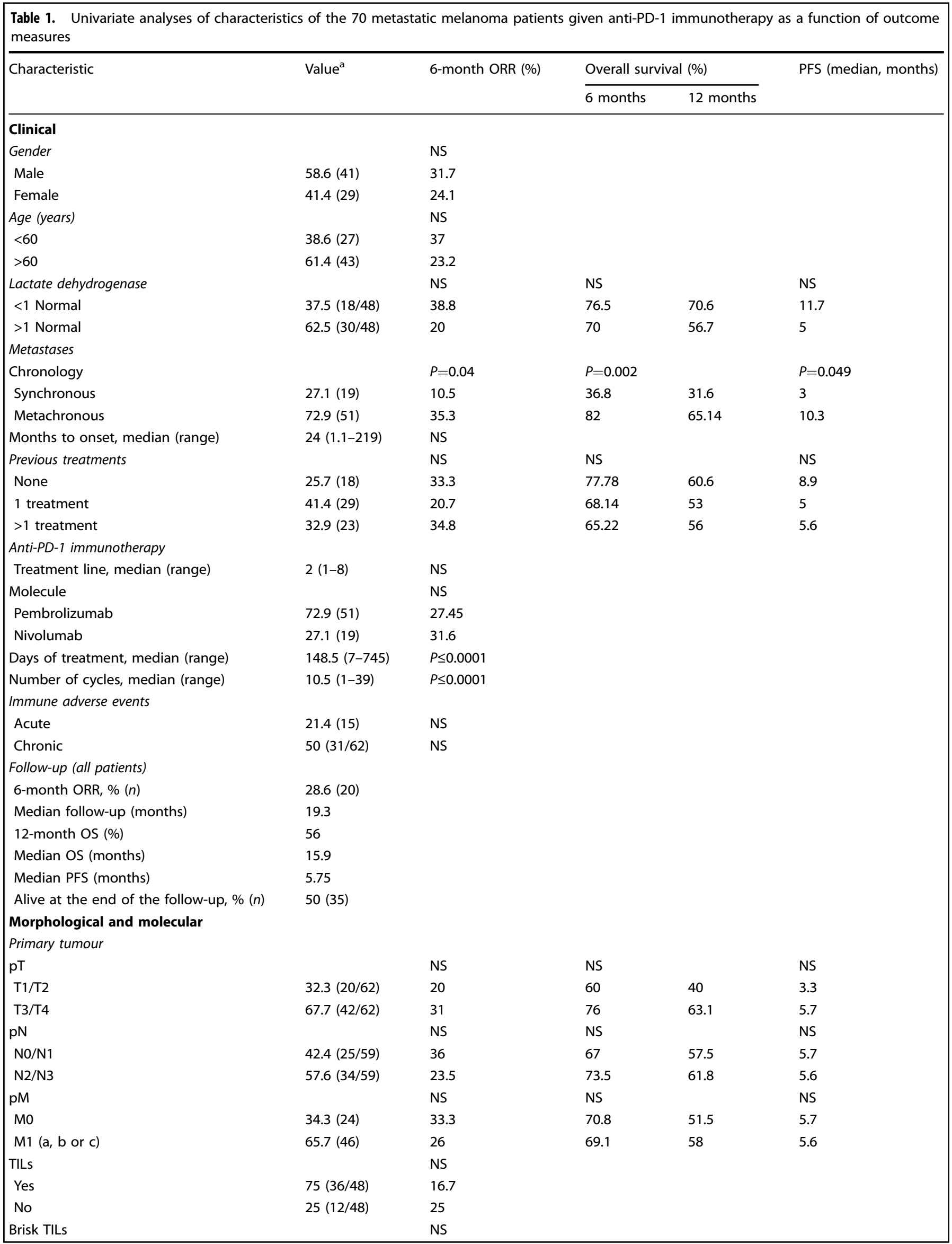


Table 1 continued

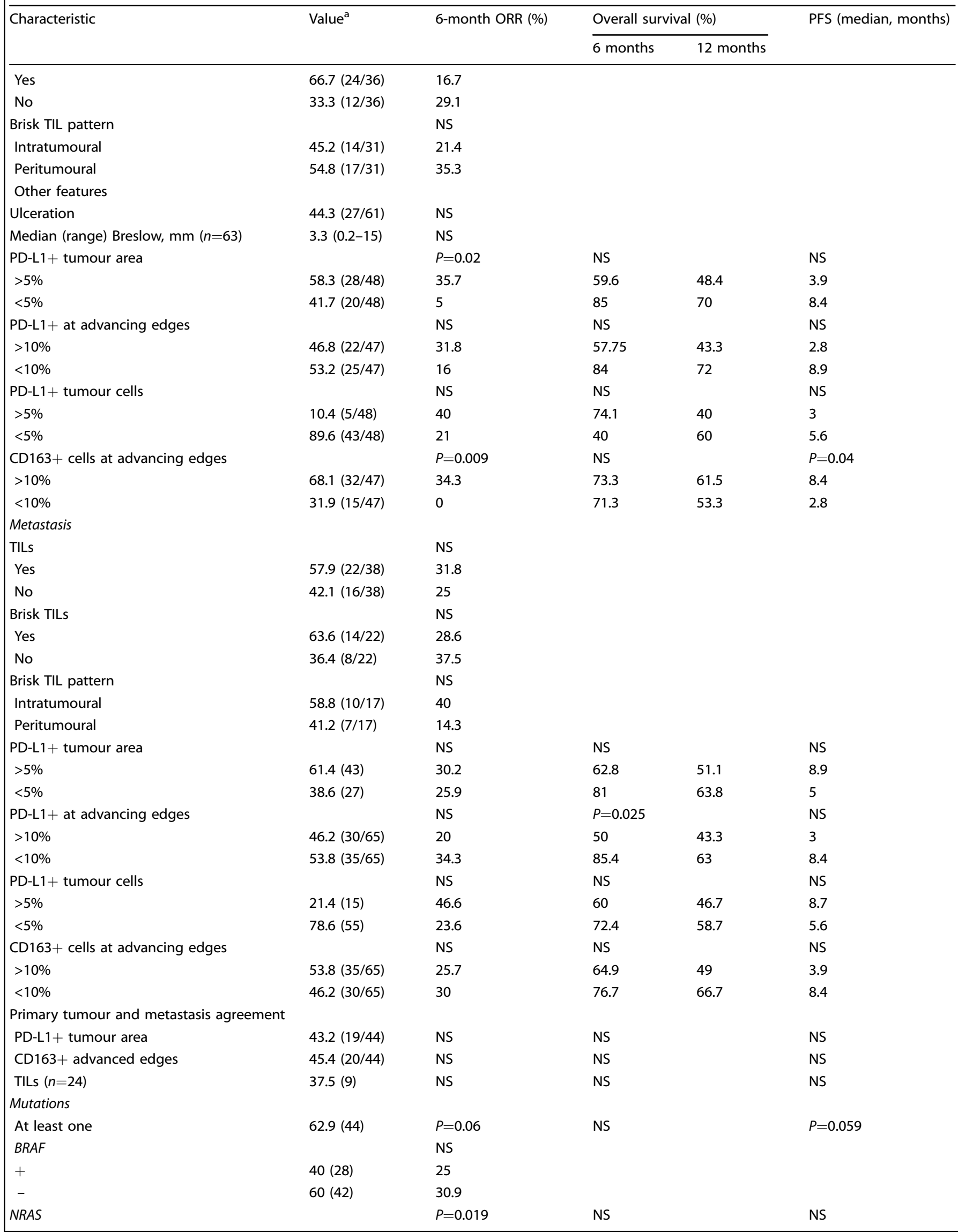




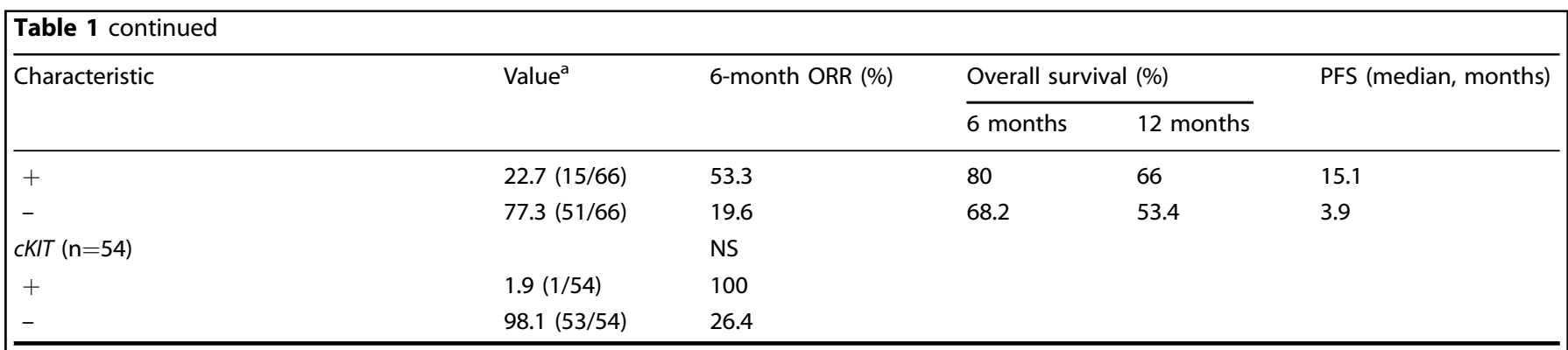

ORR objective response rate, OS overall survival, $P F S$ progression-free survival, NS non-significant: $\geq 0.05, P D-1$ programmed death-1, $P D-L 1$ programmed death-

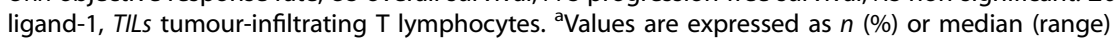

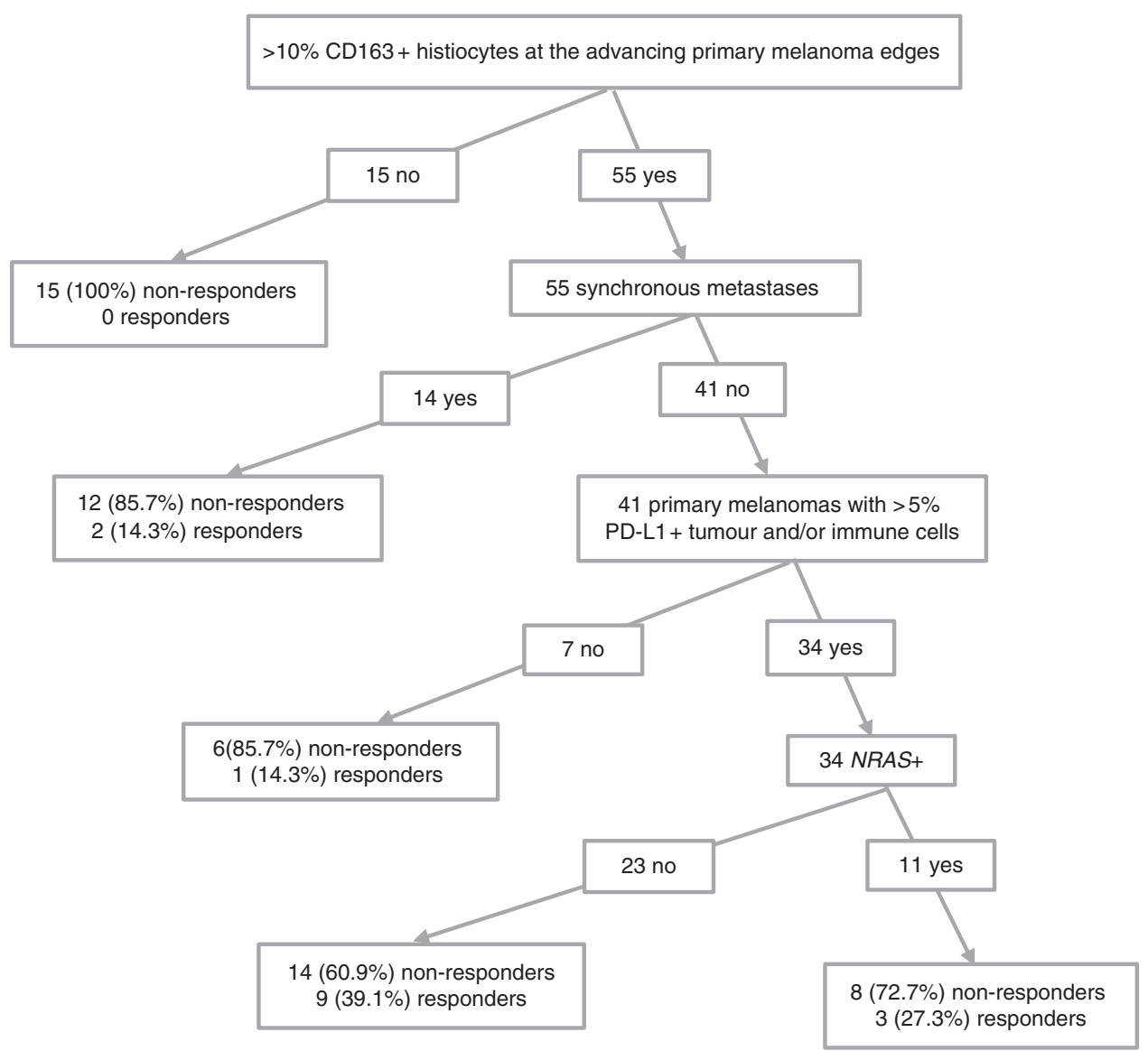

Fig. 2 Decision-tree algorithm created via recursive partitioning. This decision tree indicates how well different variables predict 6-month objective response class. Clinicians could easily use this kind of tree for routine care. PD-L1 programmed death-lig and-1

\section{RESULTS}

Seventy patients' 118 melanoma samples were examined; 48 had primary and metastatic specimens available (Table 1). Metastasis locations were: 26 in skin, 34 in lymph nodes, two in liver, two in lung and six elsewhere. Pembrolizumab was given to $72.9 \%$ of the patients and nivolumab to $27.1 \%$. Median follow-up was 19.3 (95\% confidence interval (CI): 16.2-21.4) months. The 6-month ORR was $28.6 \%$, with $69.7 \%$ (57.42-79.09\%) OS, median PFS lasted 5.75 (2.99-10.28) months, and median OS 15.9 (95\% Cl: 8.7-not reached) months. Neither IAE severity nor frequency was associated with ORR or $>5 \%$ PD$\mathrm{L} 1+$ tumour areas. Among the clinical characteristics analysed, only synchronous vs metachronous metastases differed significantly for ORR, OS and PFS (Kaplan-Meier plots available in supplementary figures 3 and 7).
At least one BRAF, neuroblastoma-RAS oncogene (NRAS) or CKIT mutation was found in $62.9 \%$ of the lesions and $22.7 \%$ were NRASmutated. Although NRAS-mutated lesions (regardless of mutation type) were associated with better ORR than those NRAS-, their corresponding OS (66\% vs $53.4 \%$ at 12 months, $P=0.32$ ) and PFS (median of 15.1 vs 3.9 months; $P=0.2$ ) were comparable. Nonsignificant trends towards better ORR and PFS were observed for patients with tumours harboring at least one mutation (Kaplan-Meier plots available in supplementary figures 4 and 8).

Labeling with the anti-PD-L1 antibodies used appeared quite similar. PD-L1-labeled areas were similar for primary melanomas and their metastases (Fig. 1). PD-L1 expression on primary melanomas, but not metastases, was associated with ORR (35.7\% for PD-L1+ vs $5 \%$ for PD-L1-; $P=0.02)$. Neither PD-L1 status at advancing edges nor the percentage of PD-L1+ tumour 
cells was associated with ORR, OS or PFS (Kaplan-Meier plots available in supplementary figures 1, 2 and 5). Agreement between primary melanoma and metastases was poor for total tumour-area PD-L1 expression, TIL assessment and CD163+ histiocytes at advancing tumour edge. None of those variables was associated with improved ORR or survival.

CD8+ TILs, seen in $75 \%$ of primary melanomas and $57.9 \%$ of metastases, were not associated with ORR or OS, regardless of the distribution pattern analysed. More than $10 \%$ of CD163+ histiocytes were observed at advancing tumour edges in $68.1 \%$ of primary melanomas and $53.8 \%$ of metastases. CD163+ expression in primary lesions was associated with better ORR and longer PFS (Kaplan-Meier plots available in supplementary figure 6). Very heterogenous TIL- and PD-L1+-cell distributions prevented subgroup assessment according to Teng's fourtier-grading. ${ }^{18}$

Interobserver reproducibility was high for PD-L1+ areas in primary melanomas and metastases (ICC $=0.86$ and 0.9 , respectively), and CD163+-histiocyte analysis at the advancing edges $(\mathrm{ICC}=0.78)$

Significant criteria $(P<0.05)$ were combined to create a decision-tree algorithm (Fig. 2), with 40\% sensitivity, 94\% specificity, $72.73 \%$ PPV and $79.66 \%$ NPV.

\section{DISCUSSION}

According to our results, PD-L1 status alone cannot be used as a reliable biomarker of therapeutic response to anti-PD-1 immunotherapy but could be combined with other criteria, e.g., synchronous metastases, $>10 \%$ CD163 + histocytes at advancing tumour edges or NRAS status (as previously reported ${ }^{19}$ ).

We observed that PD-L1 was often expressed on histiocytes closely intermingled with tumour melanocytes, which made the distinction between PDL1+ histiocytes and PD-L1+ tumour cells more difficult. The pathophysiological significance of such histiocyte PD-L1 expression remains unclear but has been reported for several cancers. ${ }^{12}$ Evaluating PD-L1-positivity on tumour and immune cells is more reliable and reproducible than tumour cells alone. Although double-labeling was not done, analysing only tumour-cell PD-L1-positivity (with a $5 \%$ cut-off) is perhaps less reliable, especially at the advancing tumour edges, where inflammatory immune cells are usually extensively intermingled. ${ }^{16}$

PD-L1 status appears to be relevant in primary melanomas. If that observation is confirmed, it could be helpful, especially because metastases are often difficult-to-access or analyse (e.g. obstacles to evaluating lymph-node metastases include intrinsic PD-L1 expression, particularly sinusal histiocytes).

To the best of our knowledge, CD163 + histiocytic infiltrates at advancing tumour edges have not yet been thoroughly evaluated in melanomas and might be a potentially helpful biomarker. CD163 + histiocytes are usually considered M2 macrophages and associated with poorer outcomes. ${ }^{14,20}$ Although surprising, our results are consistent with histiocytes playing a critical role in immune resistance.

In conclusion, identification of patient subgroups responding less well to anti-PD-1 immunotherapy with our algorithm will not avoid these agents, but might obtain better monitoring, and hence more quickly identify progressive disease. Our findings highlighted the potential biomarker role of combining CD163+ histiocytes in melanomas and their metastases with other variables but need to be validated with a larger prospective cohort, including assessment with available anti-PD-L1 monoclonal antibodies, before definitive conclusions can be drawn.

\section{ACKNOWLEDGEMENTS}

The authors thank the Centre de Ressources Biologiques/Tumour Bank of Bordeaux University Hospital and Oncopôle Toulouse for providing most samples.
Immunohistochemical technics were performed in the departments of pathology (CHU Bordeaux and Oncopole Toulouse).

\section{AUTHOR CONTRIBUTION}

Frantz Dupuis, Béatrice Vergier and Laurence Lamant designed the experiment, made the collection of tumour samples and pathological data from Bordeaux and partly Toulouse. Emilie Gerard made the collection of the clinical data from Bordeaux. Nouritza Torossian made the collection of the pathological data from Toulouse. Leonor Chaltiel and Thomas Filleron performed the statistical analysis. Marie Beylot-Barry,Caroline Dutriaux and Sorilla Prey helped write the manuscript and provided their valuable clinical advice. Nicolas Meyer helped write the manuscript, and made the collection of clinical data from Toulouse. Audrey Gros helped with the data relative to genetics and molecular biology. Marie-Laure Jullie helped with the review of the manuscript.

\section{ADDITIONAL INFORMATION}

Supplementary information is available for this paper at https://doi.org/10.1038/ s41416-018-0168-9.

Competing interests: The authors declare no competing interests.

Ethics approval and consent to participate: This study was performed in accordance with the Declaration of Helsinki. All patients provided written informed consent for the use of the samples, and the local Ethics Committee approved the study. (Ethic Committee of the university hospital of Bordeaux, reference of the case GP-CE-2018/02 attached in the cover letter).

Availability of data and materials: All data supporting the results reported in the article can be found in departments of Pathology of Bordeaux hospital (CHU) and Toulouse Oncopole.

Funding: The authors declare no funding or sponsorship (e.g. university, charity, commercial organization) for this study. Immunohistochemical technics were performed in the departments of pathology (CHU Bordeaux and Oncopole Toulouse).

Note: This work is published under the standard license to publish agreement. After 12 months the work will become freely available and the license terms will switch to a Creative Commons Attribution 4.0 International (CC BY 4.0).

\section{REFERENCES}

1. Thumar, J. R. \& Kluger, H. M. Ipilimumab: a promising immunotherapy for melanoma. Oncol. Williston Park 14, 1280-1288 (2010).

2. Larkin, J. et al. Combined nivolumab and ipilimumab or monotherapy in untreated melanoma. N. Engl. J. Med. 1, 23-34 (2015).

3. Ribas, A. et al. Pembrolizumab versus investigator-choice chemotherapy for ipilimumab-refractory melanoma (KEYNOTE-002): a randomised, controlled, phase 2 trial. Lancet Oncol. 8, 908-918 (2015).

4. Robert, C. et al. Nivolumab in previously untreated melanoma without BRAF mutation. N. Engl. J. Med. 4, 320-330 (2015).

5. Weber, J. S. et al. Nivolumab versus chemotherapy in patients with advanced melanoma who progressed after anti-CTLA-4 treatment (CheckMate 037): a randomised, controlled, open-label, phase 3 trial. Lancet Oncol. 4, 375-384 (2015).

6. Topalian, S. L. et al. Safety, activity, and immune correlates of anti-PD-1 antibody in cancer. N. Engl. J. Med. 26, 2443-2454 (2012).

7. Hamid, O. et al. Safety and tumour responses with lambrolizumab (Anti-PD-1) in melanoma. N. Engl. J. Med. 2, 134-144 (2013).

8. Abdel-Rahman, O. PD-L1 expression and outcome of advanced melanoma patients treated with anti-PD-1/PD-L1 agents: a meta-analysis. Immunotherapy $\mathbf{9}$, 1081-1089 (2016)

9. Mahoney, K. M., Freeman, G. J. \& McDermott, D. F. The next immune-checkpoint inhibitors: PD-1/PD-L1 blockade in melanoma. Clin. Ther. 4, 764-782 (2015).

10. Reck, M. et al. Pembrolizumab versus chemotherapy for PD-L1-positive non-small-cell lung cancer. N. Engl. J. Med. 19, 1823-1833 (2016).

11. Ilie, M., Hofman, V., Dietel, M., Soria, J.-C. \& Hofman, P. Assessment of the PD-L1 status by immunohistochemistry: challenges and perspectives for therapeutic strategies in lung cancer patients. Virchows Arch. Int J. Pathol. 5, 511-525 (2016).

12. Santarpia, M. \& Karachaliou, N. Tumour immune microenvironment characterization and response to anti-PD-1 therapy. Cancer Biol. Med. 2, 74-78 (2015).

13. Madore, J. et al. PD-L1 expression in melanoma shows marked heterogeneity within and between patients: implications for anti-PD-1/PD-L1 clinical trials. Pigment. Cell. Melanoma Res. 3, 245-253 (2015). 
14. Guo Q., et al. New mechanisms of tumour-associated macrophages on promoting tumour progression: recent research advances and potential targets for tumour immunotherapy. J. Immunol. Res. 2016, 9720912 (2016).

15. Hodi, F. S. et al. Long-term survival of ipilimumab-naive patients (pts) with advanced melanoma (MEL) treated with nivolumab (anti-PD-1, BMS936558, ONO-4538) in a phase I trial. J. Clin. Oncol. 15, 9002-9002 (2014).

16. Gandini, S., Massi, D. \& Mandalà, M. PD-L1 expression in cancer patients receiving anti PD-1/PD-L1 antibodies: a systematic review and meta-analysis. Crit. Rev. Oncol. Hematol. 100, 88-98 (2016).
17. Schatton, T., Scolyer, R. A., Thompson, J. F. \& Mihm, M. C. Tumour-infiltrating lymphocytes and their significance in melanoma prognosis. Methods Mol. Biol. Clifton NJ 1102, 287-324 (2014).

18. Teng, M. W. L., Ngiow, S. F., Ribas, A. \& Smyth, M. J. Classifying cancers based on Tcell infiltration and PD-L1. Cancer Res. 11, 2139-2145 (2015).

19. Johnson, D. B. et al. Impact of NRAS mutations for patients with advanced melanoma treated with immune therapies. Cancer Immunol. Res 3, 288-295 (2015).

20. Yang, L. \& Zhang, Y. Tumour-associated macrophages: from basic research to clinical application. J. Hematol. Oncol. 10, 58 (2017). 